\title{
Picosecond Absorption Spectra and Relaxation Processes of the Excited Singlet State of Pyrene in Solution
}

\author{
HIROSHI MIYASAKA, HIROSHI MASUHARA and NOBORU MATAGA \\ Department of Chemistry, Faculty of Engineering Science, Osaka University, \\ Toyonaka, Osaka 560, Japan
}

The method of analysis for the ps transient absorption spectra obtained by a ps $\mathrm{Nd}^{3+}$ : YAG laser photolysis system is described in detail, showing the results of pyrene in solution. The $S_{n} \leftarrow S_{1}$ spectrum observed immediately after excitation is rather broad and its intensity in the short wavelength region is relatively enhanced, compared to the spectrum observed at later stages. For elucidating this fast spectral change, the spectrum at $0 \mathrm{ps}$ was simulated from that at $100 \mathrm{ps,} \mathrm{evaluating} \mathrm{numerically}$ the inner filter effect, depletion of the ground state molecules and temporal characteristics of the monitoring ps continuum. The difference between the simulated and experimentally observed spectra may be ascribed to the nonlinear refractive index change of the solvent, the thermal lensing effect, or to an intrinsic relaxation of the excited molecule. Examining absorption spectra obtained under various conditions, the former two possibilities were denied for the excited pyrene system. Vibrational relaxation as well as the $S_{2} \rightarrow S_{1}$ internal conversion with a time constant of less than $10 \mathrm{ps}$ have been suggested to be responsible for this difference between the simulated and observed spectra.

\section{INTRODUCTION}

The flash photolysis method which was innovated by the pioneering work of Norrish and Porter ${ }^{1}$ has made a great contribution to the fields of chemical reaction and relaxation phenomena, and its temporal resolution has been improved to a great extent. The dynamic behavior of unstable radicals and molecular triplet states was the main subject in the $\mu$ s time range. The introduction of the $Q$-switched solid state laser and the nitrogen gas laser as an excitation light source 
made it possible to observe ns dynamics directly, and the $S_{1}$ state, with a relatively long life, as well as the $n \pi^{*}$ triplet state were studied in detail. One of the most active investigations in this ns time range is made on the electron and charge transfer phenomena in chemistry ${ }^{2}$ and $S_{1}-S_{1}$ annihilation processes in solution, molecular crystals and semiconductors. ${ }^{3}$ The ps laser photolysis method is available now and is recognized as being an indispensable technique in chemistry, physics, biology, and engineering. ${ }^{4}$

In the ps absorption measurements, molecular dynamics has been analyzed mostly by probing a transient absorption ai one wavelength. However, in general, absorption spectra of excited states and chemical intermediates overlap with each other, and their conformational change and orientational relaxation of solvent molecules result in a time-dependence of spectral band shape. For example, intramolecular exciplex systems give an absorption spectrum, the band shape of which is a function of solvent properties and delay times. ${ }^{5,6}$ Isomerization of trans-stilbene from the $S_{1}$ state, ${ }^{7}$ and vibrational relaxation of the triplet benzophenone ${ }^{8}$ as well as the excited singlet anthracene ${ }^{9}$ were proposed by analyzing absorption spectral band shapes observed immediately after excitation and at later stages. Namely, it is indispensable to measure the absorption spectra in the wide range of wavelengths for elucidating ps photophysical and photochemical processes. For this end, we have recently constructed a ps laser photolysis system with extreme reliability and high accuracy, and have applied it to molecules and molecular aggregates in solution. ${ }^{10}$

In measuring this sort of transient absorption, it is frequently observed that the absorption spectra obtained immediately after excitation are always a little different from those measured at later stages. Namely, the $S_{n} \leftarrow S_{1}$ spectrum immediately after excitation is broader than that obtained at longer delay times or its absorption intensity in the short wavelength region is relatively enhanced compared to that in the long wavelength region. It seems possible that this absorption spectral anomaly at early delay times is caused by the nonlinear refractive index change, the thermal lensing effect, the wavelength-dependence of arrival time of the ps continuum at the sample position, a transient inner filter effect due to absorption of the exciting photons by the excited states, and a depletion of the ground state molecules, etc. In other words, a computer simulation of the absorption spectra at early stages, involving various kinds of 
factors affecting the absorption measurements, is indispensable in order to obtain accurate results concerning the fast ps relaxation processes.

In this paper, time-resolved absorption spectra of pyrene and its derivatives in the $S_{1}$ state measured under various experimental conditions, using our new laser photolysis system, is reported. Several effects which may lead to spectral broadening and nonlinear dependence of absorbance on excitation intensity are demonstrated by model calculations. Comparing the measured and simulated spectra, ps relaxation processes of pyrene in the excited state are discussed.

\section{EXPERIMENTAL}

\section{Laser photolysis system}

A block diagram of the microcomputer-controlled $\mathrm{ps} \mathrm{Nd}^{3+}$ :YAG laser photolysis system is shown in Figure 1 . The output of the oscillator, mode-locked passively by a 1,2-dichloroethane solution of NDL-102 dye (Nippon Dye Lab. Co. Ltd.) was a train composed of about 12 pulses. Inside the cavity with a length of $1.2 \mathrm{~m}$, an aperture was set in order to ensure $\mathrm{TEM}_{00}$ mode. A single ps pulse extracted with Pockels cell (Laser Metrics KD*P 1072FV cell and 8601A high voltage pulse generator) was amplified in the three stages in order to give an output of $c a .30 \mathrm{~mJ}$. The distance between the first and second amplifiers was $7 \mathrm{~m}$ and a soft aperture (Hoya Electronics Co. Ltd., with a diameter giving a uniform transmission of $6 \mathrm{~mm}$ ) was set at $50 \mathrm{~cm}$ from the front of the second one. This optical arrangement guaranteed the $\mathrm{TEM}_{00}$ mode. The fundamental $(1064 \mathrm{~nm})$ pulse was partly converted to the second $(532 \mathrm{~nm})$, the third $(355 \mathrm{~nm})$, and the fourth $(266 \mathrm{~nm})$ harmonics by using type I and type II KD*P crystals of $15 \times 15 \times 15 \mathrm{~mm}$ (INRAD Co. Ltd.), and their combinations. The output energy of these beams was adjusted to be in the range of $0.2 \sim 2.5 \mathrm{~mJ}$ and their pulse width was $20 \sim 25 \mathrm{ps}$. The maximum repetition rate of this laser, including amplifiers, is 10 pps. A monitoring ps continuum for transient absorption measurements was produced by focusing the remaining fundamental pulse into a quartz cell of $10 \mathrm{~cm}$ length with a lens of $15 \mathrm{~cm}$ focal length. The material for generating the ps continuum was $\mathrm{D}_{2} \mathrm{O}$ (Merck UVASOL, $99.95 \%$ ). 


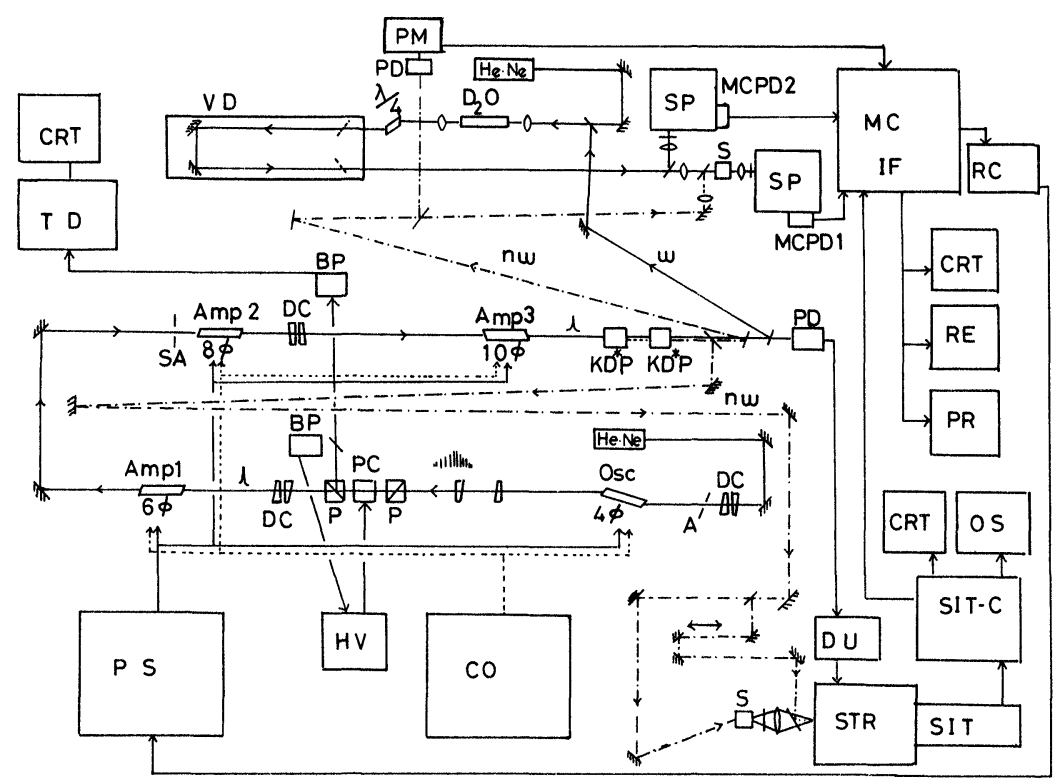

FIGURE 1 Schematic diagram of a ps $\mathrm{Nd}^{3+}$ :YAG laser photolysis system. DC; dye cell, A; aperture, P; polarizer, PC; Pockels cell, BP; biplanar phototube, SA; soft aperture, PS; power supply, HV; high voltage generator, CO; water cooler, PD; photodiode, $\mathrm{PM}$; power meter, TD; transient digitizer, VD; variable delay, SP; spectrograph, S; sample, MC; microcomputer, IF; interface, RC; remote control, RE; recorder, PR; printer, DU; delay unit, STR; streak camera, SIT; SIT camera, SIT-C; SIT camera controller, OS; oscilloscope.

Recently we have clarified the temporal characteristics of this continuum by analyzing the two-dimensional format of their streak images. ${ }^{11}$ The pulse width is $20 \sim 25$ ps which shows almost no dependence on the wavelength. The ps continuum has a distribution of arrival time at the sample position, and the blue part is delayed to the red. In Figure 2, the wavelength-dependence of this arrival time of the continuum is shown. The effect of polarization of this monitoring beam in absorption spectroscopy was avoided by leading it through a $\lambda / 4$ plate.

Excitation and monitoring beams entered the sample cell collinearly with a $1-\mathrm{cm}$ path length. The overlapping of both beams was ascertained through a $30-\mathrm{cm}$ optical path. The diameter of the excitation pulse at the front part of the cell was $2.5 \mathrm{~mm}$, the center of which 


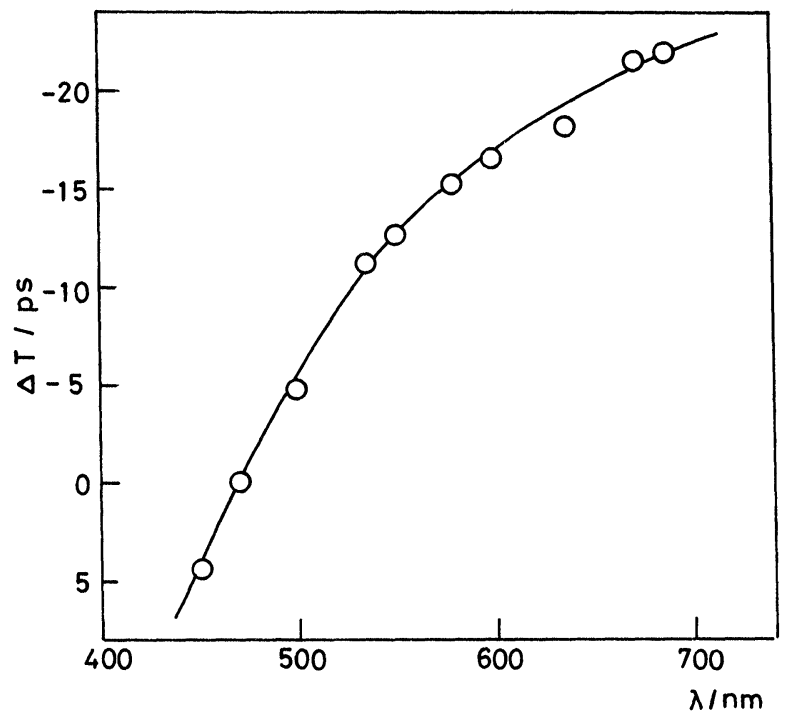

FIGURE 2 Wavelength-dependence of arrival time of the $\mathrm{D}_{2} \mathrm{O}$ ps continuum at the sample position. The time when the $470 \mathrm{~nm}$ light arrives at the sample is tentatively defined as the origin of the time scale.

was monitored by a ps continuum with a diameter of less than $1 \mathrm{~mm}$. The spectrum of the ps continuum was detected by a multichannel photodiode array (MCPD) (Matsushita Model MEL 1024KV). The principle of the present double-beam method for transient absorption measurement is the same as used before in this laboratory. ${ }^{6}$ Two MCPDs equipped with cylindrical quartz lenses are attached to spectrographs $(f=25 \mathrm{~cm}$, Jovin Yvon grating 300 lines $/ \mathrm{mm})$, covering a wavelength range of $380 \mathrm{~nm}$. The outputs of MCPD1 and MCPD2 (512 channels) in the $m$ th measurement with an excitation pulse to the sample cell are denoted by $I_{1}^{E}(m)$ and $I_{2}^{0}(m)$, respectively. They are replaced by $I_{1}^{0}(n)$ and $I_{2}^{0}(n)$ in the $n$th measurement without excitation. Because of the nonlinear property of the ps continuum generation, $I_{1}^{0}(m), I_{2}^{0}(m)$ and $I_{1}^{E}(m)$ depend on $m$, while $I_{1}^{0}(m) / I_{2}^{0}(m)$ does not change from shot to shot. Therefore, the transient absorbance is calculated as

$$
\begin{aligned}
A & =\log \left(I_{1}^{0}(m) / I_{1}^{E}(m)\right) \\
& =\log \left(I_{2}^{0}(m) / I_{1}^{E}(m)\right)-\log \left(I_{2}^{0}(n) / I_{1}^{0}(n)\right) .
\end{aligned}
$$


If we block the excitation pulse also at the $m$ th measurement, Eq. (1) should give zero. In this case the result is called a base line which is used as a reference for judging the optical alignment. One example is given in Figure 3A.

The detection system is coupled to a Sord M223 Mark II microcomputer, including a $64 \mathrm{~KB}$ memory, two floppy disks with $350 \mathrm{~KB}$, a Sord SLP-150T printer, and 12-bit A/D and D/A converters. The laser oscillation is fired also by this microcomputer. All the computer programs for controlling the system and data processing were developed in this laboratory. Laser oscillation and a single pulse extraction are monitored with a biplanar photodiode (Hamamatsu R1193U-01, -02) and Tektronix transient digitizer R7912. The excitation intensity is detected by a hand-made power meter whose digital
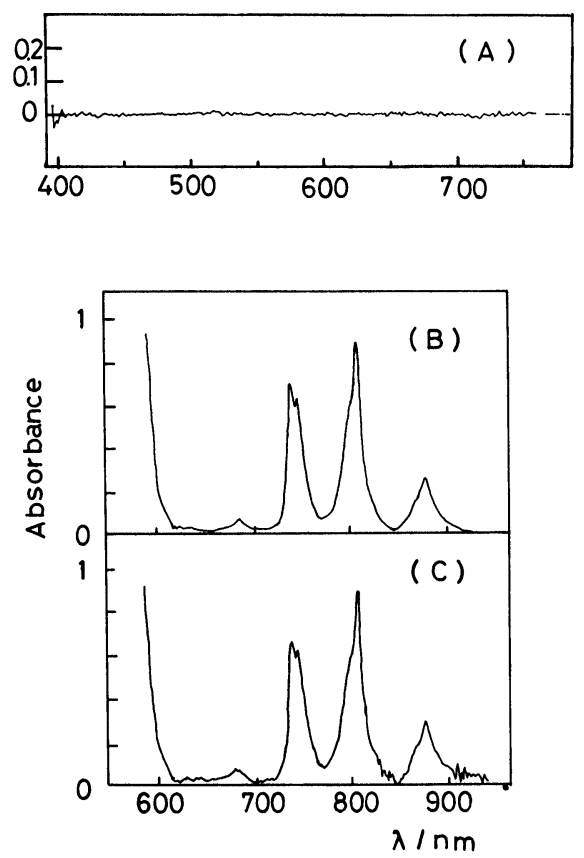

FIGURE 3 A base line of the present laser photolysis system which was obtained by averaging over 8 shots. See text. Absorption spectra of Hoya V-10 Didymium filter measured with a conventional spectrophotometer (b) and with the present laser photolysis system (c). 
output is calibrated by a Molectron J3-05DW power meter. The intensity scatter of excitation and monitoring beams are checked automatically with stringent acceptance criteria. Transient absorption spectra were obtained by averaging over 4 or 8 measurements and no smoothing was applied.

The absorption spectrum of the Hoya V-10 Didymium filter was measured by blocking the excitation beam and by comparing the ps continuum spectra obtained with and without this filter. As shown in Figure 3, the observed spectrum is in good agreement with that measured with a conventional spectrophotometer. All these results indicate that the present system is in a satisfactory condition, and its performance is summarized in Table I.

\section{Materials}

Pyrene, 1-ethylpyrene, and 2,7-di-t-butylpyrene were purified by repeated recrystallization and sublimation. Cyclohexane, $n$-hexane, liquid paraffin (Dotaito Spectrosol) were used without further purification. Since we have confirmed no effect of the dissolved oxygen upon the observed transient absorption spectra, most of the measurements were made by using aerated solutions. The concentration of pyrene was $-6.7 \times 10^{-3} \mathrm{M}$ and that of 1-ethylpyrene and 2,7di- $t$-butylpyrene was adjusted to give an absorbance of $1.0 \sim 1.8$ at $355 \mathrm{~nm}$. All the measurements were performed at room temperature $\left(25^{\circ} \mathrm{C}\right)$.

TABLE I

The performance of the microcomputer-controlled $\mathrm{ps} \mathrm{Nd}^{3+}$ :YAG laser photolysis system

\begin{tabular}{lc}
\hline Measurable wavelength range & $410 \sim 950 \mathrm{~nm}$ \\
Wavelength resolution & $3 \mathrm{~nm}$ \\
Measurable time range & $0 \sim 5 \mathrm{~ns}$ \\
Response time & $50 \mathrm{ps}$ \\
Maximum sensitivity & $0.01(\mathrm{absorbance})$ \\
Time required for measurements $^{\mathrm{b}}$ & $3 \mathrm{~min}$ \\
\hline
\end{tabular}

\footnotetext{
${ }^{a}$ Rise time from $10 \%$ to $90 \%$ of the maximum plateau value.

b Time for measuring one spectrum averaged over 8 shots and for recording it on a sheet.
} 


\section{RESULTS}

The transient absorption spectra of pyrene in cyclohexane obtained by exciting with the $355 \mathrm{~nm}$ pulse are shown in Figure 4 . The spectral band shape observed at $100 \mathrm{ps}$ is practically the same as the one reported which was assigned to the $S_{n} \leftarrow S_{1}$ transition. ${ }^{12-14}$ The origin of the time-axis in our measurements was determined as follows: the peak absorbance at $468 \mathrm{~nm}$ of the $S_{n} \leftarrow S_{1}$ spectrum of pyrene was plotted against the optical delay. Since the $S_{1}$ state of pyrene shows no decay in the 100 ps time range, there exists a plateau region in absorbance. The time when the absorbance is one half of the plateau is defined as the origin of the time-axis. If there is no contribution

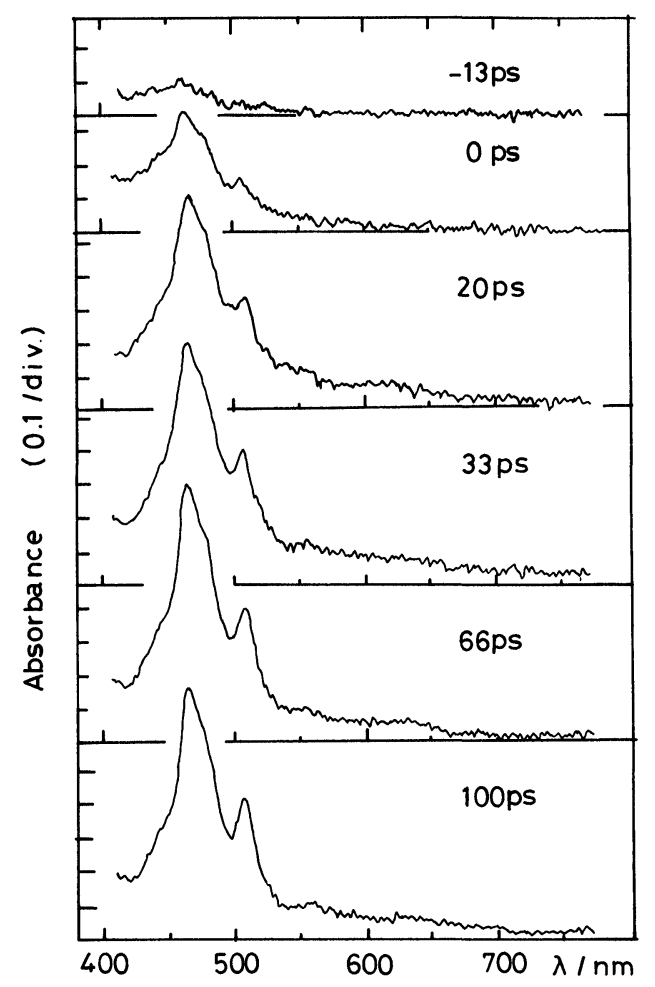

FIGURE 4 Transient absorption spectra of pyrene in cyclohexane obtained by exciting with the $355 \mathrm{~nm}$ pulse. 
of the factors which will be discussed later to the absorption spectral shape and absorption rise curve, this origin corresponds to the time when the excitation and monitoring pulses take maximum temporal overlap.

At $0 \mathrm{ps}$ two peaks at $468 \mathrm{~nm}$ and $510 \mathrm{~nm}$ are observed and the absorbance at $420 \mathrm{~nm}$ is about half of that at $468 \mathrm{~nm}$. The spectrum increases up to $66 \mathrm{ps}$ accompanied by a sharpening of the band shape, and no spectral evolution was observed at the later stages until the excimer formation was detected. Some spectra normalized at $468 \mathrm{~nm}$ are shown in Figure 5. Compared with the spectrum at 100 ps, the absorption tail below $460 \mathrm{~nm}$ is higher and a hollow at $490 \mathrm{~nm}$ is shallower at 0 ps. It is clearly demonstrated that the absorption spectral band shape depends on the delay time, and its change in the wavelength region below $468 \mathrm{~nm}$ disappears more rapidly than that in the long wavelength region above $520 \mathrm{~nm}$. Almost the same behavior was observed in the case of 1-ethylpyrene and 2,7-di-tbutylpyrene in cyclohexane.

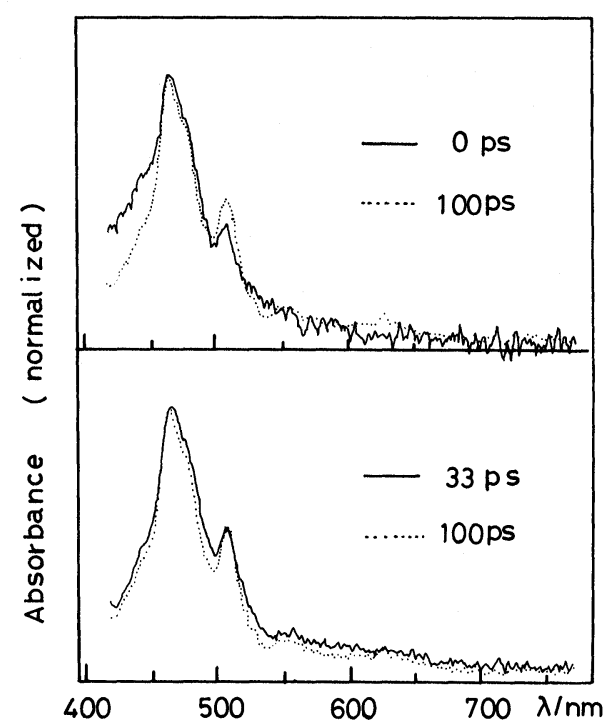

FIGURE 5 The normalized transient absorption spectra of pyrene in cyclohexane obtained by exciting with the $355 \mathrm{~nm}$ pulse. 
Excitation wavelength dependence of the present time-dependent spectral change was examined by comparing the above results with a series of time-resolved spectra obtained in the $266 \mathrm{~nm}$ photolysis. The results were confirmed to be common to both excitation wavelengths.

The transient absorption spectra of pyrene in liquid paraffin were measured by excitation with the $355 \mathrm{~nm}$ pulse. The spectra normalized at the peak of $471 \mathrm{~nm}$ are given in Figure 6 whose dependence on the delay time is almost the same as that in cyclohexane. This result, showing no solvent viscosity effect, is consistent with the depolarized property of the monitoring beam. Therefore, the present spectral anomaly at early stages has nothing to do with the rotational diffusion of the excited pyrene.

The excitation intensity effect on the transient absorption spectra was examined by the $355 \mathrm{~nm}$ photolysis of pyrene in cyclohexane. The spectra obtained at 0 ps is very sensitive to the optical alignment because the effective diameter of the excitation pulse is decreased at

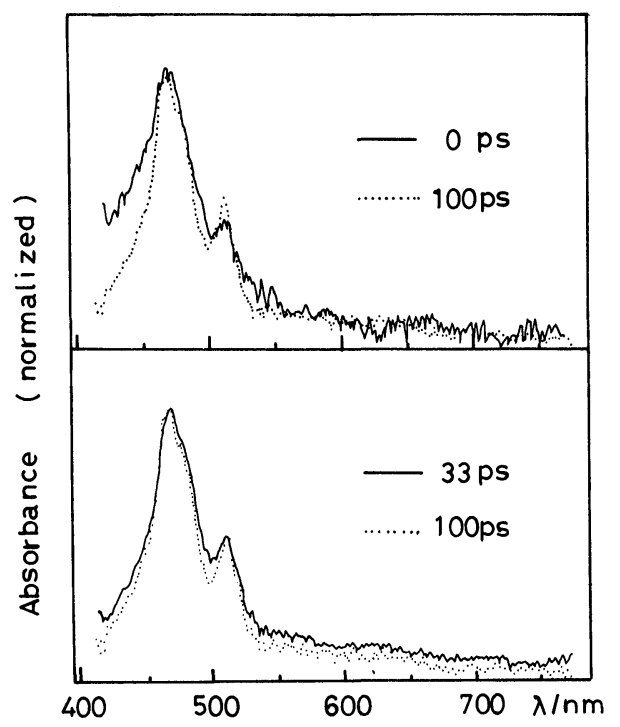

FIGURE 6 The normalized transient absorption spectra of pyrene in liquid paraffin obtained by exciting with the $355 \mathrm{~nm}$ pulse. 
early stages compared to that of other delay times. It is worth noting that the present measurements were performed under experimental conditions adjusted with significant care. In Figure 7, absorption spectra observed by changing the excitation intensity are given by normalizing the spectra at the $468 \mathrm{~nm}$ peak. The spectral shape at each delay time is confirmed to be independent of the excitation intensity within experimental errors. The effect of the excitation intensity on the absorption rise curve at $468 \mathrm{~nm}$ was examined. It was demonstrated that the rise curve is almost independent of the excitation intensity.

The absorbance of the $468 \mathrm{~nm}$ band at $100 \mathrm{ps}$ is plotted against the excitation intensity in Figure 8. Although a linear relation is expected, a saturation tendency was observed even in the range of low excitation intensity.

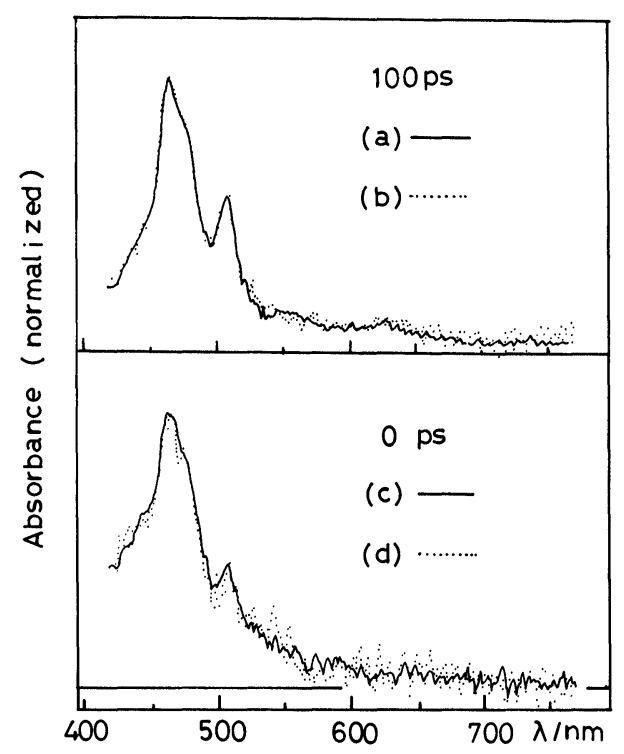

FIGURE 7 Excitation intensity dependence of the normalized transient absorption spectra of pyrene in cyclohexane. The excitation wavelength is $355 \mathrm{~nm}$. Excitation intensity focused into the spot of $3.8 \times 10^{-2} \mathrm{~cm}^{2}$ is (a) $2.12 \mathrm{~mJ}$, (b) $0.25 \mathrm{~mJ}$, (c) $1.24 \mathrm{~mJ}$, and (d) $0.24 \mathrm{~mJ}$ which give (a) 1.12 , (b) 0.33 , (c) 0.36 , and (d) 0.17 as the absorbance at $468 \mathrm{~nm}$. 


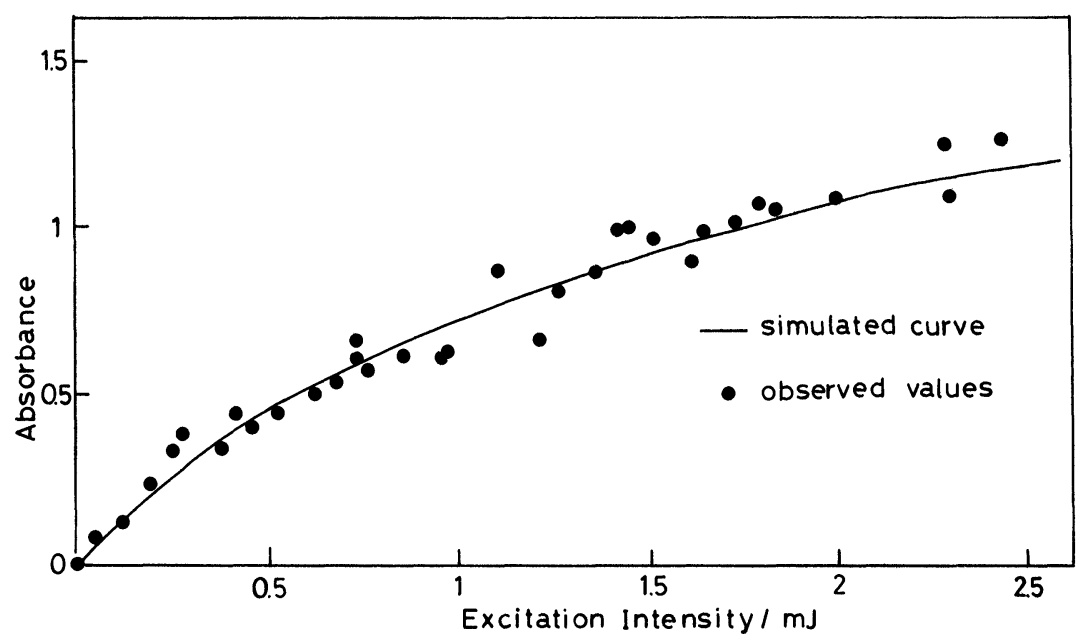

FIGURE 8 Excitation intensity dependence of the $S_{n} \leftarrow S_{1}$ absorbance of pyrene in cyclohexane at $468 \mathrm{~nm}$. The delay time is $100 \mathrm{ps}$. The theoretical curve ( $\longrightarrow$ ) is the same as in Figure 12. (see text.)

\section{GENERAL CONSIDERATIONS ON TRANSIENT ABSORPTION SPECTRA}

In general, it is a rather difficult problem to obtain a reliable and accurate transient absorption spectrum and its rise and decay curves, using the laser photolysis method. The nonlinear absorption behavior due to high excitation intensity, which has been familiar in ns laser photolysis studies, will also be involved in ps spectroscopy. Furthermore, owing to the extremely high intensity of a ps exciting pulse, it may be necessary to take into account some additional nonlinear factors for a correct interpretation of the observed spectra. Examining the results of our systematic investigations on the ns and ps transient absorption spectra, the phenomena affecting the absorption spectral measurements can be summarized as follows:

1) A spatial stray light effect. The monitoring beam which passes the unexcited volume acts as a stray light for transient absorption spectroscopy. ${ }^{15}$

2) A depletion effect on the ground state molecule. If the excitation conditions are such that most of the molecules in the ground state 
are excited, the concentration of the excited species would not increase appreciably with increasing excitation intensity. Lachish et al. reported a qualitative criterion for this depletion effect as $\sigma h \geqslant 1$, where $\sigma$ and $h$ are the absorption cross-section in $\mathrm{cm}^{2}$ and the excitation intensity in photons $\mathrm{cm}^{-2}$, respectively. ${ }^{16,17}$

3) A transient inner filter effect. The excitation pulse may be absorbed by the excited singlet state or by other laser-induced chemical transients, suppressing the effective number of excitation photons. ${ }^{17,18}$

4) An effect of the pulse width on the excitation and monitoring beam. Since the FWHM-value of the $355 \mathrm{~nm}$ pulse and the $\mathrm{D}_{2} \mathrm{O}$ ps continuum are measured to be 22 and $24 \mathrm{ps,} \mathrm{respectively,}{ }^{11}$ the temporal overlapping of both pulses should be considered for elucidating the absorbance obtained at early delay times. Some parts of the monitoring continuum which arrives at the sample position before the excitation beam act as a stray light in absorption spectroscopy. This contribution can be evaluated by the usual convolution method.

5) An effect of a wavelength-dependent distribution of arrival time on the ps continuum. According to our recent study, ${ }^{11}$ the ps continuum of $\mathrm{D}_{2} \mathrm{O}$ used here has a distribution of arrival time at the sample position and the blue part is delayed compared to the red part.

6) A nonlinear refractive index change. Since the excitation photon density per unit area per unit time is very high, a change of the refractive index of solvents may be induced. ${ }^{19}$ Namely, an excitation pulse produces a non-uniform distribution of the refractive index which leads to the scattering of the ps continuum, resulting in an apparent transient absorption.

7) A thermal lensing effect. A similar refractive index change is also induced by local heating around the absorbing molecule. ${ }^{20,21}$

8) Two-photon excitation of solvents. The ps $355 \mathrm{~nm}$ photolysis of aromatic liquids and solvents containing double bonds gives $S_{n} \leftarrow S_{1}$ absorption spectra with appreciable intensity, ${ }^{22,23}$ which may modify the absorption spectral shape.

9) Resonance Raman scattering due to solvents. In the case of aliphatic solvents, the Stokes-Raman scattering induced by the $355 \mathrm{~nm}$ pulse disturbs the absorption spectral measurements. ${ }^{22}$ 
All these phenomena are considered to induce an apparent spectral change at early delay times, and to affect the absorption rise curve. Therefore, it is indispensable to examine experimentally or to evaluate numerically these effects in order to investigate fast relaxation processes on the basis of the transient absorption spectral data. Among the above described phenomena, effects (8) and (9) can be excluded by selecting appropriate solvents, and increasing the solute concentration. The effect of spatial stray light (1) can also be decreased to a great extent by adjusting the optical alignment carefully. The contribution of effects (2) and (3) in transient absorbance can be estimated numerically if molar extinction coefficients of the ground and excited states at the excitation wavelength are known. Concerning the effect (4), measured pulse widths of the excitation and monitoring beams make it possible to convolute the rise curve of the transient absorbance. Using information on the effect (5), the transient absorption spectra at different delay times can be simulated from the above convoluted rise curves at various wavelengths. If there still exists a difference between the experimentally observed and numerically simulated spectra at early delay times, it can be ascribed to the phenomena (6), (7) or an intrinsic fast relaxation process. Along this line we examine the present $S_{n} \leftarrow S_{1}$ absorption spectra of pyrene in cyclohexane.

\section{COMPUTER SIMULATION OF TRANSIENT ABSORPTION SPECTRA}

\section{Simulation method}

Using a computer, NEC ACOS 6 System 900 of this University, we demonstrate the important contributions of effects (2), (3) and (4) and simulate the early stage absorption spectral shape from the spectrum observed at $100 \mathrm{ps}$. In the following we summarize the assumptions used in the simulation.

1) The spatial stray light effect is completely neglected.

2) The excitation beam and the monitoring continuum at each wavelength have a Gaussian profile along the time axis, and the Gaussian variances are calculated from the FWHM-value which are almost independent of the wavelength and were determined with streak camera measurements. ${ }^{11}$ 
3) Both pulses have the same velocity.

4) Vibrational relaxation and any other processes are rapidly com pleted and no spectral change occurs in the ps time region. Namely, molar extinction coefficients of the excited pyrene are not a function of time.

5) The sample cell with a 1-cm path length is composed of a number of thin layers for which the Lambert-Beer equation holds. This assumption may be necessary even in the case of the thinner sample cell (for example, a $0.1-\mathrm{cm}$ thickness), since the solute concentration may be increased in the latter case in order to give an appropriate absorbance at the excitation wavelength.

6) An absolute delay time is defined as the time difference between maxima in the time-profile of both pulses. Namely, the origin of the time scale is the time when the excitation and monitoring pulses take maximum temporal overlap.

Now, the functions of excitation and monitoring pulses are given by Eq. (2) and (3), respectively:

$$
\begin{aligned}
\Phi(t) & =\Phi_{\mathrm{tot}} \cdot(2 \pi)^{-0.5} \sigma_{e}^{-1} \exp \left(-t^{2} / 2 \sigma_{e}^{2}\right) \\
I(t, \mu) & =I_{\mathrm{tot}} \cdot(2 \pi)^{-0.5} \sigma_{m}^{-1} \exp \left(-(t-\mu)^{2} / 2 \sigma_{m}^{2}\right)
\end{aligned}
$$

Here, $\Phi_{\text {tot }}$ and $I_{\text {tot }}$ are the total photon numbers of the excitation pulse and of the monitoring continuum at a certain wavelength, respectively. The corresponding Gaussian variances are $\sigma_{e}^{2}$ and $\sigma_{m}^{2}$, and $\mu$ is an absolute delay time defined in the assumption (6).

For numerical calculation, the cell with a $1-\mathrm{cm}$ path length was spatially divided into 100 layers of $0.01-\mathrm{cm}$ thickness and the functions given as Eqs. (2) and (3) are deemed to be composed of many sections of $1 \mathrm{ps}$ width. Using the Lambert-Beer equation for thin layers, the following equations are derived for the relations between concentrations of the excited and ground state molecules in each layer at different times.

$$
\begin{aligned}
C_{e}^{l}\left(t_{i}\right)= & C_{e}^{l}\left(t_{i-1}\right)+\Phi^{l-1}\left(t_{i}\right)\left\{1-10^{-B^{l}\left(t_{i-1}\right) \Delta l}\right\} \\
& \times \varepsilon_{g, 355} C_{\mathrm{g}}^{l}\left(t_{i-1}\right) /\left\{B^{l}\left(t_{i-1}\right) N_{a v}^{\prime} \Delta V\right\} \\
B^{l}\left(t_{i-1}\right)= & \varepsilon_{g, 355} C_{\mathrm{g}}^{l}\left(t_{i-1}\right)+\varepsilon_{e, 355} C_{e}^{l}\left(t_{i-1}\right) \\
C_{\mathrm{g}}^{l}\left(t_{i}\right)+C_{e}^{l}\left(t_{i}\right)= & C_{0}
\end{aligned}
$$


where, $C_{e}^{l}\left(t_{i}\right)$ is the concentration of the excited molecule in the $l$-th layer at the time $t_{i} . C_{\mathrm{g}}^{l}\left(t_{i}\right)$ is the concentration of the ground state molecule in the $l$-th layer at the time $t_{i}$. $C_{0}$ is total concentration of the molecule. $\varepsilon_{g, 355}$ and $\varepsilon_{e, 355}$ are molar extinction coefficient of the ground and the excited state molecules at $355 \mathrm{~nm} . \Phi^{l-1}\left(t_{i}\right)$ is the number of the excitation photons incident to the $l$-th layer at the time $t_{i} . B^{l}\left(t_{i-1}\right)$ is the total absorbance for the $l$-th layer due to the ground and the excited state molecules at the time $t_{i-1} .1000 N_{\mathrm{av}}^{\prime}$ is Avogadro's number. $\Delta l$ is the thickness of layers. $\Delta V$ is the effective total volume of the sample cell in $\mathrm{cm}^{3}$ where the excited states are produced.

Neglecting an inner filter effect and depletion of the ground state molecules, this $\Delta V$ was calculated as the first approximation to Eq. (7).

$$
\Delta V=\left(\varepsilon_{e, p} / A_{e, p}(100 \mathrm{ps})\right)\left(\Phi_{\mathrm{tot}}\left(1-10^{-\varepsilon_{\mathrm{g}, 355}} C_{\mathrm{o}}\right) / N_{\mathrm{av}}^{\prime}\right)
$$

where, $A_{e, p}$ and $\varepsilon_{e, p}$ are the observed absorbance and the molar extinction coefficient of the excited state at wavelength $p$, respectively. Using a computer, $C_{e}^{l}\left(t_{i}\right)$ in Eq. (4) was successively calculated from $l=1$ to $l=100$. The intensity of the monitoring beam is decreased from $l=1$ to $l=100$, according to the following equation,

$$
I^{l}\left(t_{j}, \mu\right)=I^{l-1}\left(t_{j}, \mu\right) \cdot 10^{-\varepsilon_{e, p}} l_{e}^{l}\left(t_{i}\right) \Delta l
$$

The monitoring intensity at the time $t_{j}$ incident to the $l$-th layer is given by $I^{l-1}\left(t_{j, \mu}\right)$. Through the absolute delay time $\mu, t_{i}$ and $t_{j}$ are related to each other. Now the transient absorbance can be calculated according to Eq. (9).

$$
A_{e, p}(\mu)=\log \left(I_{\text {tot }} / \sum_{t_{j}=1}^{t_{\text {max }}} I^{101}\left(t_{j}, \mu\right)\right)
$$

A simple numerical evaluation of transient absorbance and its dependence on molar extinction coefficients, excitation intensity, and excitation as well as monitoring pulse widths were given in this way. However, the errors produced by neglecting an inner filter effect and depletion of the ground state molecules in the estimation of $\Delta V$ may be significant if the corresponding molar extinction coefficients are large and excitation intensity is high. In such a case, a more reliable $\Delta V$-value was numerically evaluated by substituting the calculated $A_{e, p}(100 \mathrm{ps})$ into Eq. (7). This iterative calculation converged to a definite value of $\Delta V$ and, consequently, an improved $A_{e, p}(\mu)$. 


\section{Simulated results}

First we demonstrate the importance of taking into consideration the effect (4), namely, the pulse width of excitation and monitoring beams. At the very early delay times, some parts of the latter beam arrive at the sample position earlier than the excitation pulse and operate like a stray light. This leads to a nonlinear relation between transient absorbance and its molar extinction coefficient, since the relative contribution of this stray light in the transmitted intensity of the monitoring beam increases as the molar extinction coefficient becomes larger. Since pyrene is studied as an example in this work, $\varepsilon_{g, 355}$ and $\varepsilon_{e, 468}$ are 300 and $16800,{ }^{13}$ respectively. Because the inner filter effect was not involved in this demonstration, $\varepsilon_{e, 355}$ was set to 0 . Referring to the experimental results, absorbances of $A_{e, 468}(100 \mathrm{ps)}$ and $A_{g, 355}$ before excitation were assumed to be 1 and 2, respectively, from which the $\Delta V$ value was obtained by Eq. (7). In this case, no iterative procedure was applied. Calculated relations between absorbance at 0 ps and the molar extinction coefficient are given in Figure 9 as a function of the monitoring pulse width. In the ideal case where the monitoring pulse shape is a delta-function, a linear relation was

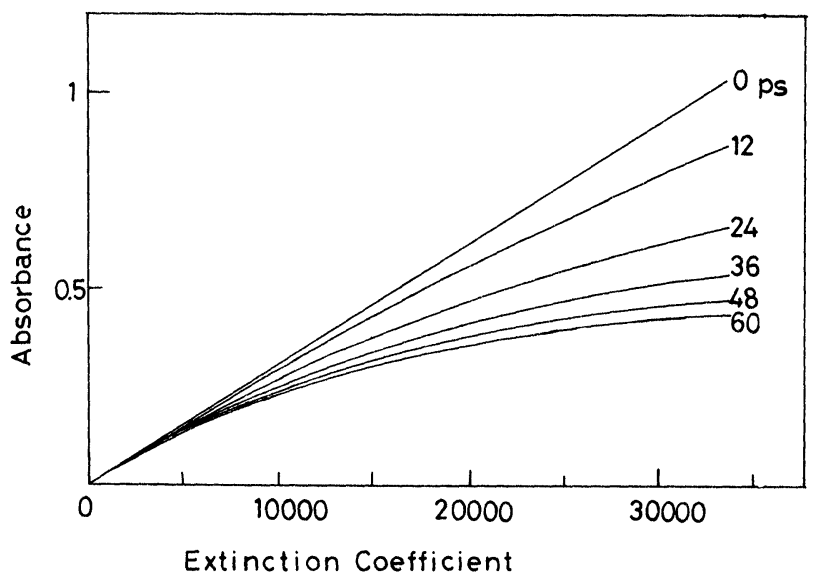

FIGURE 9 Calculated relations between the $S_{n} \leftarrow S_{1}$ absorbance at 0 ps and the corresponding molar extinction coefficient. The notation $0 \mathrm{ps}$ means that the monitoring pulse shape is a delta-function and other numbers represent the FWHM-value of the ps continuum. Used parameters were set as follows; $\varepsilon_{g, 355}=300, \varepsilon_{e, 468}=16800$, $\varepsilon_{e, 355}=0, A_{\mathrm{g}, 355}=2$, and $A_{e, 468}$ at $100 \mathrm{ps}=1 . \Delta V$ was evaluated by Eq. (7). See text. 
observed, while a saturation tendency increases as the FWHM-value does.

Similarly, the dependence of the rise curve of the $S_{n} \leftarrow S_{1}$ absorbance on the molar extinction coefficient at the probing wavelength was examined. A clear demonstration is given in Figure 10 where the pulse width of the ps continuum was set to the measured value of $24 \mathrm{ps}^{11}$ The normalized rise curves show that the rise of the absorbance with high $\varepsilon_{e, 468}$ is delayed compared to that with low $\varepsilon_{e, 468}$, although the $S_{1}$ state is always produced immediately after excitation. An uncertainty in the rise time evaluated as a difference of times where absorbances become $10 \%$ and $90 \%$ of the maximum absorbance, respectively, is shown to be several ps, and the time when absorbance is half of the maximum value is possibly shifted by $\pm 5 \mathrm{ps}$. Namely, formation rate constants of chemical intermediates at these very early stages cannot be determined without data on $\varepsilon_{e, p}$.

Secondly, we show the numerical evaluation of the depletion effect on the value of $A_{e, 468}(100 \mathrm{ps})$ of pyrene. In order to simplify the calculation, an inner filter effect was neglected, namely, $\varepsilon_{e, 355}=0$. $A_{e, 468}(100 \mathrm{ps}), A_{g, 355}$ and $\varepsilon_{e, 468}$ are the same as those used in the above simulations except $\varepsilon_{8,355}$ which is now a variable parameter.

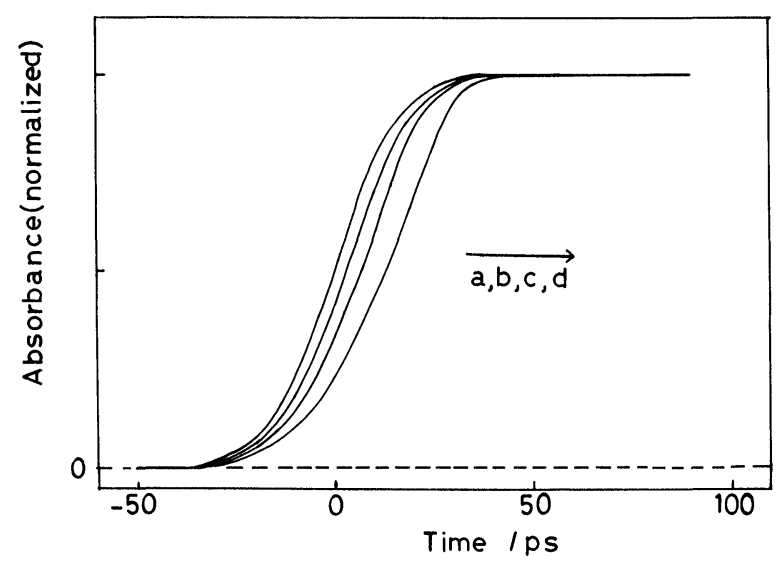

FIGURE 10 Calculated rise curves of the $S_{n} \leftarrow S_{1}$ absorbance as a function of the corresponding molar extinction coefficient. All the curves are normalized at the plateau value. $\varepsilon_{e, 468}$ is (a) 2100 , (b) 16800 , (c) 33600 and (d) 67200 . Other parameters are the same as given in Figure 9. 
The $\Delta V$-value was evaluated by the iterative procedure, and the calculated results are shown in Figure 11. In the case of $\varepsilon_{8,355}=300$, deviation from the linear relation was very small, while saturation behavior was observed when $\varepsilon_{g, 355}$ was increased. In the plateau region, most of the solute molecules are in the excited state and the latter concentration is no longer dependent on excitation intensity. The rise curve is also affected and becomes steep as the depletion of the ground state molecules is increased. In this case an apparently faster rate constant of formation of the excited state would be obtained.

In Figure 12, the inner filter effect due to the excited singlet state is displayed. The value of $\varepsilon_{g, 355}$ was set to 300 , which does not result in an appreciable depletion effect as shown in Figure 11. The value of $A_{e, 468}(100 \mathrm{ps})$ increases, parallel to excitation intensity in the case of $\varepsilon_{e, 355}=0$, while the saturation tendency becomes larger as the

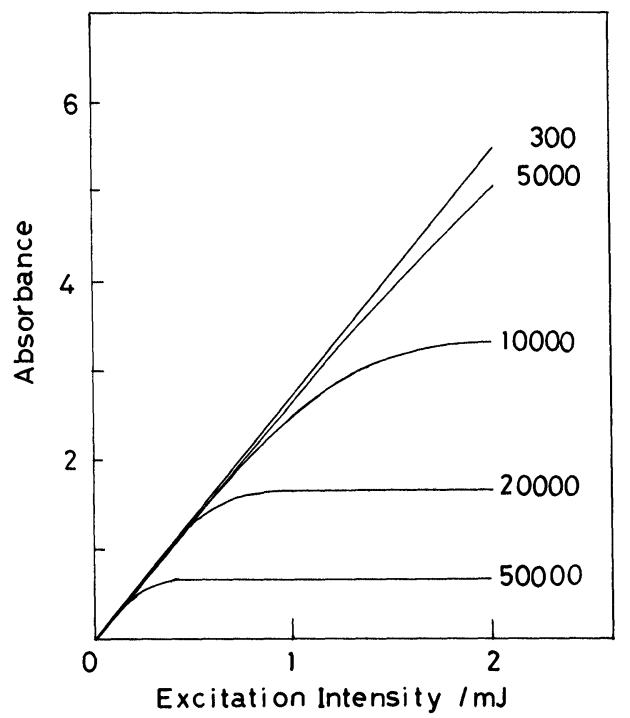

FIGURE 11 Simulated depletion effect on the $S_{n} \leftarrow S_{1}$ absorbance $(468 \mathrm{~nm})$ of pyrene at $100 \mathrm{ps}$. The numbers in the Figure are $\varepsilon_{\mathrm{g}, 355}$, and other parameters except $\varepsilon_{e, 355}=21100$ are the same as given in Figure 9. The iterative procedure was applied for evaluating the $\Delta V$ value (See text below Eq. (9)). Although the vertical scale is given for the pyrene having $\varepsilon_{e, 468}=16800$, saturation behavior will be observed in the lower transient absorbance for molecules with smaller $\varepsilon_{e, p}$. 


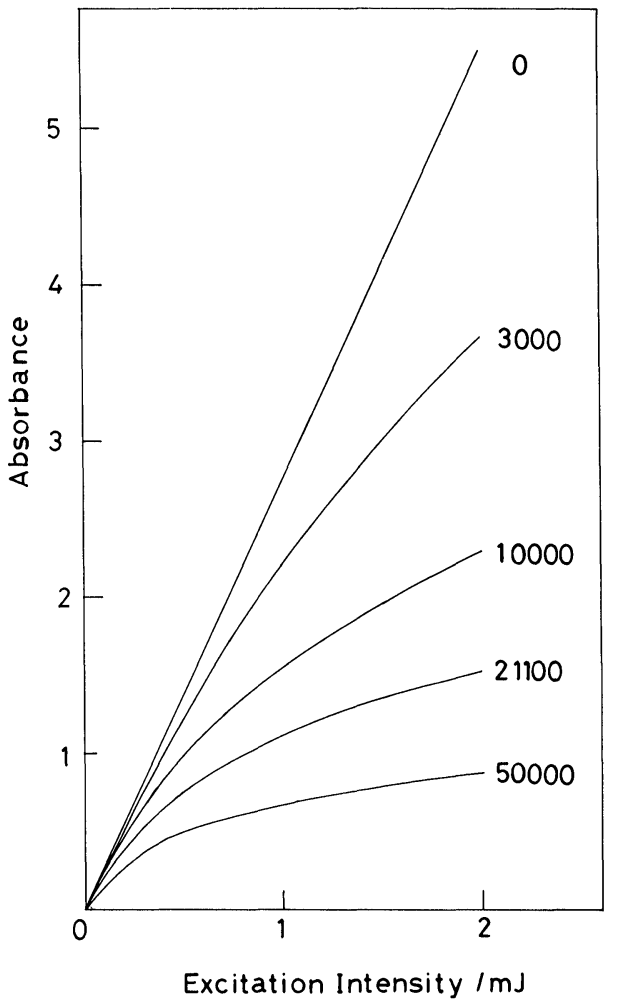

FIGURE 12 Simulated inner filter effect on the $S_{n} \leftarrow S_{1}$ absorbance $(468 \mathrm{~nm})$ of pyrene at $100 \mathrm{ps}$. The numbers in the Figure are $\varepsilon_{e, 355}$, and other parameters are the same as given in Figure 11. The interative procedure was applied for evaluating the $\Delta V$ value (see text below Eq. (9)). Although the vertical scale is given for the pyrene having $\varepsilon_{e, 468}=16800$, saturation behavior will be observed in the lower transient absorbance for molecules with smaller $\varepsilon_{e, p}$.

latter value is increased. It is worth noting that pyrene has an $\varepsilon_{e, 355}$ of 21100 and it is indispensable to take into consideration this inner filter effect. The simulated curve with $\varepsilon_{e, 355}=21100$ is compared with the experimental result in Figure 8 . The satisfactory agreement between both curves indicates that a nonlinear dependence of this $S_{n} \leftarrow S_{1}$ band on excitation intensity is mainly ascribed to the inner filter effect. The $\Delta V$-value evaluated here by the iterative procedure gives the value of the excitation beam diameter, which is in accord 
with that determined directly. Therefore, the present simulation approach for transient absorption measurements is meaningful. In addition, the excitation intensity dependence of the $S_{n} \leftarrow S_{1}$ rise curve was also simulated, the result of which shows that the rise becomes steeper as the contribution of the inner filter effect increases.

Since the importance of effects (2) (4) in transient absorption measurements is numerically demonstrated here, now we can compare the simulated and the experimental rise curves. As shown in Figure 13 , a good agreement between both curves is observed for the $S_{n} \leftarrow S_{1}$ band of pyrene in cyclohexane, which can be explained as follows. The contribution of the depletion effect is small in the present $355 \mathrm{~nm}$ photolysis of pyrene, while the rise becomes steep because of the inner filter effect. However, the latter effect may be cancelled by an apparently delayed rise since the absorbance with rather high $\varepsilon_{e, 468}$ is monitored with the ps continuum having a finite pulse width. As described in the RESULTS, the origin of the time-axis has been empirically determined by plotting the $S_{n} \leftarrow S_{1}$ absorbance of pyrene at $468 \mathrm{~nm}$, which is now confirmed to be quite reasonable by the present theoretical simulation.

In a similar way, the rise curve of the pyrene $S_{n} \leftarrow S_{1}$ absorption can be simulated at each wavelength, since its molar extinction

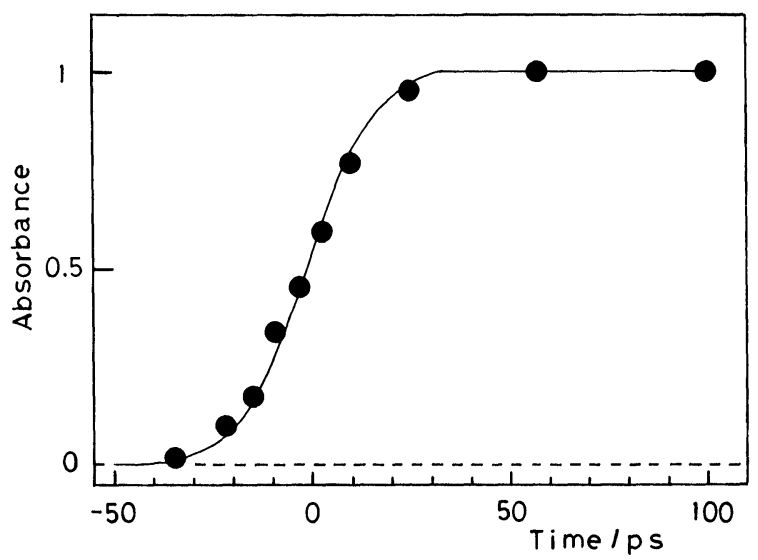

FIGURE 13 Simulated rise curve (-) of the $S_{n} \leftarrow S_{1}$ absorbance of pyrene in cyclohexane at $468 \mathrm{~nm}$. Used parameters are as follows; $\varepsilon_{g, 355}=300, \varepsilon_{e, 355}=21100$, and $\varepsilon_{e, 468}=16800$. Experimental rise curve (O) was obtained by the $355 \mathrm{~nm}$ photolysis. 
coefficient is reported. ${ }^{13}$ Plotting the calculated absorbance at $0 \mathrm{ps}$ against the wavelength, the simulated $S_{n} \leftarrow S_{1}$ spectra were obtained. As shown in Figure 14a and b, the spectrum at 0 ps simulated by involving the effects $(2) \sim(4)$ is only a little broader than that measured at $100 \mathrm{ps}$, and quite different from the one at 0 ps. This is considered to be ascribed to the effect (5). Therefore, calculated rise curves at each wavelength were shifted along the time-axis, referring to the distribution function of arrival time of the ps continuum shown in Figure 2. This gives the simulated absorption spectrum at $0 \mathrm{ps}$ which is compared with the spectra measured at 0 and 100 ps in Figure $14 \mathrm{c}$ and d.

The difference shown in Figure $14 \mathrm{c}$ corresponds to the contribution of effects $(2) \sim(4)$ since the simulation spectra are based on the one measured at $100 \mathrm{ps}$. This simulated spectrum is compared with the experimental one at $0 \mathrm{ps}$ in Figure 14d. We do not discuss here the

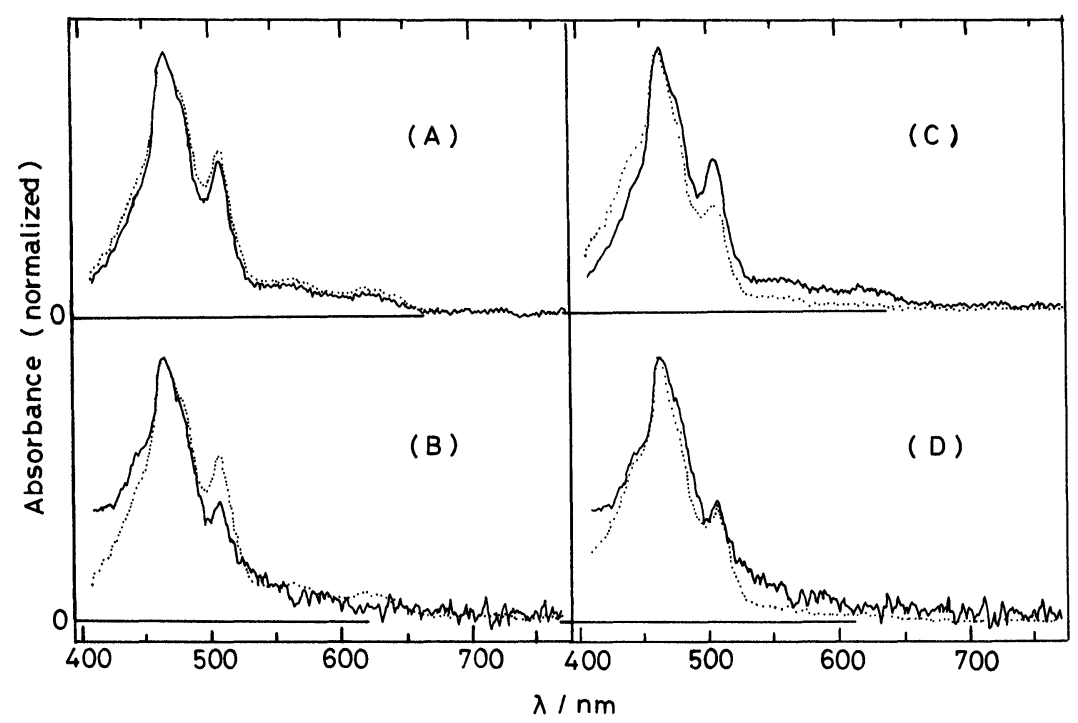

FIGURE 14. Simulated and measured transient absorption spectra of the excited singlet state pyrene in cyclohexane. (a) measured at $100 \mathrm{ps}(\stackrel{)}{\longrightarrow}$ and simulated at 0 ps by considering the effects $(2) \sim(4)(\cdots)$. (b) measured at $0 \mathrm{ps}(-)$ and simulated at $0 \mathrm{ps}$ by considering the effects $(2) \sim(4)(\cdots)$. (c) Measured at $100 \mathrm{ps}(-)$ and simulated at $0 \mathrm{ps}$ by considering the effects $(2) \sim(5)(\cdots)$. (d) measured at $0 \mathrm{ps}(\longrightarrow)$ and simulated at 0 ps by considering the effects $(2) \sim(5)(\cdots)$. 
difference observed below $450 \mathrm{~nm}$, since the $S / N$ ratio in this wavelength region is poor because of the very weak intensity of the ps continuum around this region. On the other hand, the differences observed above $450 \mathrm{~nm}$ are recognized to be larger than the experimental error, in view of the better $S / N$ ratio in this region. Therefore, it is concluded that the difference between the observed and simulated spectra in Figure 14d is physically significant and spectral characteristics at early delay times are summarized as follows: the shoulder observed just above $468 \mathrm{~nm}$ and the band around $540 \mathrm{~nm}$ are intensified.

\section{DISCUSSION}

We have simulated the ps transient absorption spectrum of the excited pyrene by considering various effects which modify the spectrum and its rise curve. The difference between the simulated and measured spectra at 0 ps (see Figure 14d) may be due to the effect (6), (7) or a ps relaxation process, all of which are not involved in the present simulation. First, we consider the effect (6) as a possible contribution to the nonlinear refractive index. In general, the refractive index of materials is a function of the electric field, which may be written as

$$
n=n_{0}+n_{2}\left\langle E^{2}\right\rangle+\cdots
$$

where $\left\langle E^{2}\right\rangle$ is the time average of the square of the instantaneous electric field. ${ }^{19}$ A non-uniform distribution of this refractive index induced by a high-intensity ps pulse results in a lens-like effect which may scatter some parts of the monitoring continuum. If the scattered beam deviates from the propagating axis and does not enter the MCPD detectors, an apparent absorption spectrum overlapped on the intrinsic one will arise. In the opposite case a stray light is involved in the monitoring optical path, suppressing the absorption intensity. The spatial and temporal characteristics of the nonlinear index change was calculated by Sala and Richardson. ${ }^{24}$ The index change is induced by electric hyperpolarizability and orientation anisotropy in the molecular polarizability. The former polarizability responds in time to the change of incident laser pulse intensity, while the change of the latter is delayed compared to the applied field. Whether or not this refractive index change of the solvent affects the absorption 
spectral shape can be examined by investigating the excitation intensity dependence of the transient absorption spectra. As shown in Figure 7, transient absorption spectra observed at 0 and $100 \mathrm{ps}$ are almost independent of the excitation intensity. This experimental result denies the contribution of the fast and delayed index change of refraction in absorption measurements.

The effect (7) of a thermal lensing phenomenon is another possible cause leading to the present difference between the simulated and measured spectra at 0 ps. The excess energy between the excitation level and the relaxed excited state is released to the surrounding solvents and forms a non-uniform distribution of the refractive index affecting the transient absorption measurements. However, its formation time-constant is considered to be given as the beam radius divided by the speed of sound in liquids, ${ }^{20,21}$ and the rise of this effect in the monitoring area of $20 \sim 30 \mu \mathrm{m}$ was measured to be a few tens ns. ${ }^{21}$ Since this estimation gives $1 \mu \mathrm{s}$ or longer for the present optical arrangement, it is difficult to ascribe the present result to the thermal lensing effect. This conclusion is supported by our recent study on the 1,2,4,5-tetracyanobenzene-dimethylsulfoxide charge-transfer complex in acetonitrile. ${ }^{25}$ The yield of ionic photodissociation to 1,2,4,5-tetracyanobenzene anion and dimethylsulfoxide cation is negligible, and most of the excitation energy is considered to be converted rapidly to thermal energy. Even in this case no transient absorption in the wavelength range of $420 \sim 800 \mathrm{~nm}$ was observed from 33 ps to $5 \mathrm{~ns}$ delay times, and was in good agreement with the base line.

Because we have simulated the contribution of inner filter and depletion effects, the pulse width and the wavelength-dependence of the monitoring ps continuum, and have excluded the nonlinear index change of refraction and thermal lensing effect as a probable explanation, the difference between the simulated and measured spectra at 0 ps (see Figure 14d) is concluded to be due to some intrinsic relaxation processes. First we examine the possibility that the vibrational relaxation process is the $S_{1}$ state results in this spectral change from 0 to $100 \mathrm{ps}$. The first direct demonstration of this sort of vibrational relaxation in the excited electronic state in solution was given for the triplet benzophenone by Greene et $a .^{8}{ }^{8}$ The triplet-triplet absorption spectra of benzophenone measured by a $\mathrm{Nd}^{3+}$ glass laser photolysis method showed a change during the first 50 ps from the excitation 
pulse. The spectrum measured at early stages is broadened and shifted to longer wavelengths compared to those at longer delay times. This behavior is consistent with theoretical model calculations. ${ }^{26}$ Anderson reported that this vibrational relaxation was also observed for the excited singlet state anthracene in 3-methylpentane. ${ }^{9}$ Such a characteristic spectral broadening and its change was more clearly demonstrated in the gas phase experiments, where such change occurs more slowly. ${ }^{27,28}$ Before considering this vibrational relaxation for the present system, the $S_{n} \leftarrow S_{1}$ absorption spectrum of pyrene should be assigned. As already mentioned, the spectrum measured here is quite similar to those observed before ${ }^{12-14}$ and summarized in Table II where theoretical results by PPP SCF-CI calculation ${ }^{13}$ are also listed. The absorption peaks at 468 and $508 \mathrm{~nm}$ correspond to the $S_{11} \leftarrow S_{1}$ and $S_{10} \leftarrow S_{1}$ transition, respectively, and there is no calculated transition which can be assigned to the shoulder just above $468 \mathrm{~nm}$. The energy separation between the peak at $468 \mathrm{~nm}$ and this shoulder is several hundred $\mathrm{cm}^{-1}$ which is close to the vibrational interval observed in the $S_{1} \leftarrow S_{0}$ band. The relative intensity of this shoulder to the maximum at $468 \mathrm{~nm}$ is larger at $0 \mathrm{ps,} \mathrm{compared} \mathrm{to}$ that of the simulated spectra. This behavior is quite similar to that observed in the triplet benzophenone and the excited singlet anthracene, although the present vibrational structure is rather diffuse. From this viewpoint of vibrational relaxation in the excited state, the fact that the weak band at $540 \mathrm{~nm}$ is observed at $0 \mathrm{ps}$ can also be explained as follows. There are four transitions whose oscillator strengths are zero in the corresponding wavelength range. If the molecules are in the higher vibrational states, anharmonicity will increase, leading to a larger vibronic coupling. Through this interaction, the forbidden transition, for example $S_{9} \leftarrow S_{1}$, will obtain its intensity. Although this spectral change is rather small, its relaxation time can be estimated to be several ps. This is a reasonable value which is consistent with the data on the relaxation rate constant of the excited and ground state molecules. Recently, Choi et al. obtained a value of $15 \mathrm{ps}$ for the vibrational relaxation time of the excited 3,4,9,10-dibenzopyrene by the consecutive two-photon excitation and frequency up-conversion methods. ${ }^{29}$ Laubereau et al. studied the vibrational relaxation process in the ground state by measuring the stimulated anti-Stokes Raman scattering of vibrationally excited liquids. ${ }^{30}$ For $\mathrm{CHCl}_{3}, \mathrm{CH}_{3} \mathrm{CH}_{2} \mathrm{OH}$ and related molecules, this 


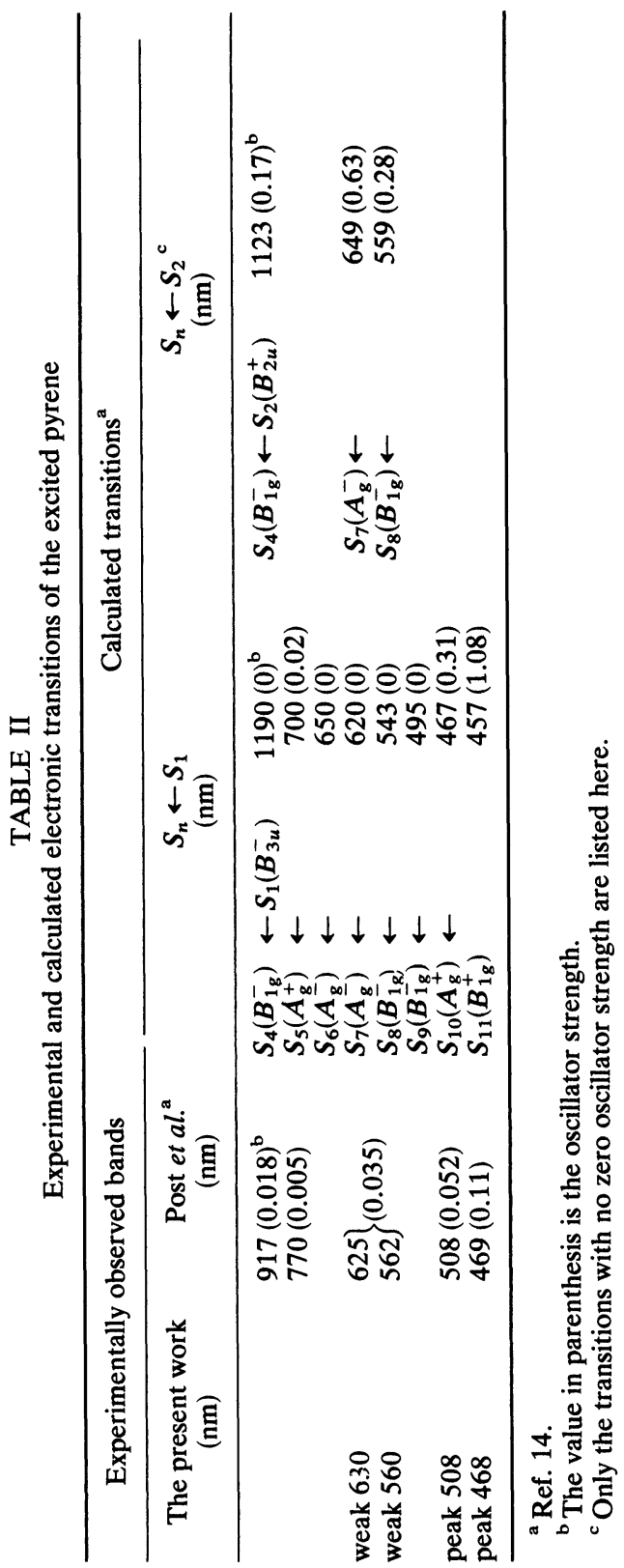


relaxation occurs via one or two exponential decay processes in the time range of $1 \sim 100 \mathrm{ps}$. This behavior was interpreted in terms of mode-matching, rotational coupling, Fermi-resonance coupling and Coriolis interaction. The present analysis on the excited pyrene, together with previous reports, ${ }^{8,9,28}$ implies that the vibrationally unrelaxed state should be considered as an active state for energy, electron and proton transfers.

An alternative mechanism for the present early spectral change immediately after excitation is to consider the internal conversion from the $S_{2}$ to $S_{1}$ states, in view of the fact that the fluorescence from the $S_{2}$ state is observed in the gas-phase pyrene. ${ }^{31,32}$ In the $266 \mathrm{~nm}$ photolysis, pyrene is excited to the $S_{3}$ state which will relax to $S_{1}$ through $S_{2}$. There may be a possibility also that the $355 \mathrm{~nm}$ pulse irradiates the tail of the $S_{2} \leftarrow S_{0}$ transition. If the relaxation from $S_{2}$ to $S_{1}$ states is slow enough to be detected in the present laser photolysis, the observed spectral change near 0 ps delay time can be explained. Namely, the $S_{n} \leftarrow S_{2}$ absorption spectrum may be overlapped with the $S_{n} \leftarrow S_{1}$ bands at early stages and its contribution decreases rapidly. The theoretical consideration indicates that $S_{7} \leftarrow S_{2}$ and $S_{8} \leftarrow S_{2}$ transitions with moderate intensity will be observed in the wavelength region above $510 \mathrm{~nm}$, as shown in Table II. On the other hand there are no absorption bands predicted in the range of $400 \sim 500 \mathrm{~nm}$. The sharp $S_{11} \leftarrow S_{1}$ band below $500 \mathrm{~nm}$ becomes strong and the bands above $500 \mathrm{~nm}$ due to the $S_{7} \leftarrow S_{2}$ and $S_{8} \leftarrow S_{2}$ transitions disappear as the $S_{2} \rightarrow S_{1}$ internal conversion is completed. These theoretical predictions are consistent with the present ps spectral change observed around $540 \mathrm{~nm}$, while the spectral difference at the shoulder just above $468 \mathrm{~nm}$ cannot be explained. Therefore both the $S_{2} \rightarrow S_{1}$ internal conversion and the vibrational relaxation in the $S_{1}$ state may be responsible for the ps spectral change.

\section{CONCLUDING REMARKS}

We have presented in this paper a general method to analyze the ps transient absorption spectra of molecules in solution measured with a $\mathrm{Nd}^{3+}$ : YAG laser photolysis system. It has been demonstrated that the simulation of the absorption spectrum observed immediately after excitation is indispensable for elucidating fast ps relaxation processes. 
The present method of analysis is considered to be quite general and can be applied to other ps and also sub-ps laser photolysis studies.

At present, for ps transient absorption spectral measurements, the following three methods are available in addition to the present mode-locked solid-state laser photolysis. (1) The light pulse from the mode-locked $\mathrm{cw}$ dye laser and its second harmonic are used for excitation and the monitoring ps continuum light was generated by focusing the amplified original pulse into $\mathrm{H}_{2} \mathrm{O}$ or $\mathrm{D}_{2} \mathrm{O} .^{33}$ (2) The transient species produced by the mode-locked $\mathrm{cw}$ dye laser are detected with a tunable cw probe laser gated by a sub-ps frequency up-conversion. ${ }^{34}$ (3) A $\mu$ s flash lamp was used as a monitoring light source and its ps intensity change due to transient absorption was detected by a streak camera. ${ }^{35}$ As shown in this paper, the temporal characteristic of the ps continuum should be made clear for elucidating the transient absorption spectral change at early stages. It is worth noting that a distribution of its arrival time at the sample position is wider than the FWHM-value of the original $1064 \mathrm{~nm}$ pulse. ${ }^{11}$ Therefore, there might be a possibility that the monitoring continuum produced by a few ps $\mathrm{Nd}^{3+}$ : glass laser and sub-ps dye laser has a wider pulse width than the corresponding original pulses, which results in the same problem considered here. The effect of the pulse width of a monitoring light is important also in the method (1), and inner filter and depletion effects should be examined for numerical evaluation of the results obtained by all these methods. These procedures are necessary for the quantitative evaluation of the ps relaxation process from the results of transient absorption spectral measurements. We have demonstrated this idea by measuring and simulating the transient absorption spectral change of the excited pyrene.

\section{Acknowledgement}

The authors thank Dr. Noriaki Ikeda for his experimental help and discussions. N.M. and H.M. thank the Japanese Ministry of Education, Science, and Culture for financial support through a Grant-in-Aid for Special Project Research on Photobiology.

\section{References}

1. Fast Reactions and Primary Processes in Chemical Kinetics, ed. S. Claesson (Almqvist and Wiksell, Uppsala, 1967). 
2. N. Mataga and M. Ottolenghi, Molecular Association, Vol. 2, ed. R. Foster (Academic Press, London, 1979) Chapter 1, pp. 1-78; N. Mataga, Molecular Interactions, Vol. 2, ed. H. Ratajczak and W. J. Orville-Thomas (John Wiley and Sons Ltd., London, 1981) Chapter 12, pp. 509-570; H. Masuhara and N. Mataga, Accounts Chem. Res. 14, 312 (1981).

3. For example, C. E. Swenberg and N. F. Geacintov, Organic Molecular Photophysics, Vol. 1, ed. J. B. Birks (John Wiley and Sons Ltd., London, 1973) Chapter 10, pp. 489-564; D. von der Linde, Ultrashort Light Pulses ed. S. L. Shapiro (SpringerVerlag, Berlin, 1977) Chapter 5, pp. 203-273.

4. Picosecond Phenomena II ed. R. M. Hochstrasser, W. Kaiser and C. V. Shank (Springer-Verlag, Berlin, 1980).

5. N. Nakashima and N. Mataga, Chem. Phys. Lett. 35, 487 (1975); N. Nakashima, M. Murakawa and N. Mataga, Bull. Chem. Soc. Jpn. 49, 854 (1976); M. Migita, M. Kawai, N. Mataga, Y. Sakata and S. Misumi, Chem. Phys. Lett. 53, 67 (1978); N. Mataga, M. Migita and T. Nishimura, J. Mol. Struct. 47, 199 (1978).

6. T. Okada, M. Migita, N. Mataga, Y. Sakata and S. Misumi, J. Am. Chem. Soc. 103, 4715 (1981).

7. B. I. Greene, R. M. Hochstrasser and R. B. Weisman, Chem. Phys. Lett. 62, 427 (1979); F. E. Doany, B. I. Greene and R. M. Hochstrasser, Chem. Phys. Lettt. 75, 206 (1980); B. I. Greene, R. M. Hochstrasser and R. B. Weisman, Chem. Phys. 48, 289 (1980); B. I. Greene, R. M. Hochstrasser and R. B. Weisman, J. Chem. Phys. 71, 544 (1979).

8. B. I. Greene, R. M. Hochstrasser and R. B. Weisman, J. Chem. Phys. 70, 1247 (1979).

9. R. W. Anderson, Picosecond Phenomena II ed. R. M. Hochstrasser, W. Kaiser and C. V. Shank (Springer-Verlag, Berlin, 1980) p. 163.

10. H. Masuhara, N. Ikeda, H. Miyasaka and N. Mataga, J. Spectrosc. Soc. Jpn. 31, 19 (1982); M. M. Martin, N. Ikeda, T. Okada and N. Mataga, J. Phys. Chem. 86, 4148 (1982); A. Karen, N. Ikeda, N. Mataga and F. Tanaka, Photochem. Photobiol. in press.

11. H. Masuhara, H. Miyasaka, A. Karen, T. Uemiya, N. Mataga, M. Koishi, A. Takeshima and Y. Tsuchiya, Opt. Commun. 44, 426 (1983).

12. Y. Nakato, N. Yamamoto and H. Tsubomura, Chem. Phys. Lett. 2, 57 (1968).

13. M. F. M. Post, J. Langelaar and J. D. W. van Voorst, Chem. Phys. Lett. 10, 468 (1971).

14. H. Schomburg, Ph.D. Thesis, Göttingen, 1975.

15. C. R. Goldschmidt, Laser in Physical Chemistry and Biophysics, ed. J. JoussotDubien (Elsevier, Amsterdam, 1975) p. 499.

16. U. Lachish, A. Schafferman and G. Stein, J. Chem. Phys. 64, 4205 (1976).

17. H. Masuhara, S. Ohwada, N. Mataga, A. Itaya, K. Okamoto and S. Kusabayashi, J. Phys. Chem. 84, 2363 (1980); H. Masuhara, N. Tamai, K. Inoue and N. Mataga, Chem. Phys. Lett. 91, 109 (1982).

18. For example, M. M. Fischer, B. Veyret and K. Weiss, Chem. Phys. Lett. 28, 60 (1974).

19. D. H. Auston, Ultrashort Light Pulses, ed. S. L. Shapiro (Springer-Verlag, Berlin, 1977) Chapter 4.

20. D. S. Kliger, Accounts Chem. Res. 13, 129 (1980); private communication (1982).

21. K. Fuke, private communication (1982).

22. H. Masuhara, H. Miyasaka, N. Ikeda and N. Mataga, Chem. Phys. Lett. 82, 59 (1981).

23. K. Hamanoue, T. Hidaka, T. Nakayama and H. Teranishi, Chem. Phys. Lett. 82, 55 (1981). 
24. K. Sala and M. C. Richardson, Phys. Rev. A 12, 1036 (1975).

25. H. Masuhara, H. Miyasaka, T. Uemiya and N. Mataga, to be published.

26. G. R. Fleming, O. L. J. Gijzeman and S. H. Lin, J. Chem. Soc. Farad. Trans. II 70, 1074 (1974).

27. Y. Hirata and I. Tanaka, Chem. Phys. 25, 381 (1977).

28. H. Schröder, H. J. Neusser and E. W. Schlag, Chem. Phys. Lett. 54, 4 (1978).

29. K. J. Choi and M. R. Topp, Chem. Phys. Lett. 69, 441 (1980); K. J. Choi, B. P. Boczar and M. R. Topp, Chem. Phys. 57, 415 (1981).

30. A. Laubereau, S. F. Fischer, K. Spanner and W. Kaiser, Chem. Phys. 31, 331 (1978).

31. H. Baba, A. Nakajima, M. Aoi and K. Chihara, J. Chem. Phys. 55, 2433 (1971).

32. T. Deinum, C. J. Werkhoven, J. Langelaar, R. P. H. Rettschnick and J. D. W. van Voorst, Chem. Phys. Lett. 12, 189 (1971).

33. E. P. Ippen and C. V. Shank, Picosecond Phenomena, ed. C. V. Shank, E. P. Ippen and S. L. Shapiro (Springer-Verlag, Berlin, 1978) p. 103.

34. J. M. Wiesenfeld and E. P. Ippen, Chem. Phys. Lett. 67, 213 (1979).

35. M. Sumitani and K. Yoshihara, Bull. Chem. Soc. Jpn. 55, 85 (1982). 